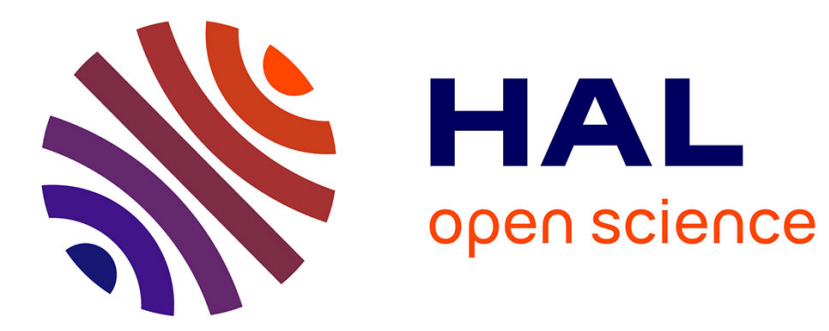

\title{
Structure of a pseudomerohedrally twinned monoclinic crystal form of a pyridoxal phosphate-dependent catalytic antibody
}

\author{
Béatrice Golinelli-Pimpaneau
}

\section{- To cite this version:}

Béatrice Golinelli-Pimpaneau. Structure of a pseudomerohedrally twinned monoclinic crystal form of a pyridoxal phosphate-dependent catalytic antibody. Acta crystallographica Section D : Structural biology [1993-..], 2005, 61, pp.472 - 476. 10.1107/s0907444905003331 . hal-03281479

\author{
HAL Id: hal-03281479 \\ https://hal.science/hal-03281479
}

Submitted on 8 Jul 2021

HAL is a multi-disciplinary open access archive for the deposit and dissemination of scientific research documents, whether they are published or not. The documents may come from teaching and research institutions in France or abroad, or from public or private research centers.
L'archive ouverte pluridisciplinaire HAL, est destinée au dépôt et à la diffusion de documents scientifiques de niveau recherche, publiés ou non, émanant des établissements d'enseignement et de recherche français ou étrangers, des laboratoires publics ou privés. 
Acta Crystallographica Section D

Biological

Crystallography

ISSN 0907-4449

Editors: E. N. Baker and Z. Dauter

\section{Structure of a pseudomerohedrally twinned monoclinic crystal form of a pyridoxal phosphate-dependent catalytic antibody}

\section{Béatrice Golinelli-Pimpaneau}

Copyright $($ International Union of Crystallography

Author(s) of this paper may load this reprint on their own web site provided that this cover page is retained. Republication of this article or its storage in electronic databases or the like is not permitted without prior permission in writing from the IUCr. 
Acta Crystallographica Section D

Biological

Crystallography

ISSN 0907-4449

\section{Béatrice Golinelli-Pimpaneau}

Laboratoire d'Enzymologie et de Biochimie Structurales, Bâtiment 34, CNRS, 1 Avenue de la Terrasse, 91190 Gif-sur-Yvette, France

Correspondence e-mail: beatrice.golinelli@lebs.cnrs-gif.fr

\section{Structure of a pseudomerohedrally twinned monoclinic crystal form of a pyridoxal phosphate- dependent catalytic antibody}

The purification, crystallization and structure determination at $2.3 \AA$ resolution of the complex of the pyridoxal-5' -phosphate (PLP) dependent catalytic antibody 15A9 with a phosphopyridoxyl-L-alanine (PPL-L-alanine) substrate analogue are described. The crystal belongs to space group $P 2_{1}$, with two molecules in the asymmetric unit related by non-crystallographic symmetry. The unit-cell parameters are $a=63.5$, $b=81.7, c=79.3 \AA$ and $\beta$ is fortuitously $90^{\circ}$. Refinement of the structure converged at unacceptably high $R$ factors. Although the traditional analysis of intensity distribution did not indicate twinning, pseudomerohedral twinning was revealed by a newer test based on local intensity differences [Padilla \& Yeates (2003), Acta Cryst. D59, 1124-1130]. When the potential twinning operator was included in SHELX, the structure could be satisfactorily refined with a twinning fraction of 0.46 , indicating a nearly perfect hemihedrally twinned crystal. One of the active sites is occupied by the phosphopyridoxyl-L-alanine ligand, while one iodide ion mimics the cofactor phosphate group in the other. Four other iodide ions are present in the structure: two are involved in specific intermolecular contacts and two dictate the conformation of the CDRH3 loop in each molecule.

\section{Introduction}

Twinning is a crystal-growth anomaly in which an ostensibly single crystal comprises separate crystal domains, the underlying lattices of which differ in orientation. True merohedral twinning only occurs in crystal systems where the twin operator is a symmetry operator of the crystal system but not of the point group of the crystal. For biological macromolecules, this happens only in crystal systems belonging to point groups 3, 32, 4, 6 and 23 (Yeates, 1997). Pseudomerohedry occurs when the twin operator belongs to a higher crystal system than the structure. In a monoclinic space group, twinning by pseudomerohedry can only occur in the case of a very special unit-cell geometry. An orthorhombic metric can be emulated with the following particular unit-cell parameters: (i) $a \simeq c$ (Yang et al., 2000), (ii) $\beta=90^{\circ}$ (Larsen et al., 2002; Barends \& Dijkstra, 2003) or (iii) the unit-cell parameters satisfy the condition $c \cos \beta=-a / 2$, i.e. the two monoclinic cells of the twin domains have coincident $a$ and $b$ axes with opposite directions and can be superimposed by a twofold rotation about an axis perpendicular to $a$ and $b$. This twofold axis runs parallel to the $b$ axis of the emulated orthorhombic unit cell, which is the $c^{*}$ direction of the monoclinic cell, leading to the twin law $(-h,-k, h+l)$ (Declercq \& Evrard, 2001; Rudolph et al., 2004). Two of these examples are the only reported cases of pseudomerohedral twinning in a monoclinic space group mimicking a primitive orthorhombic space group
Received 13 November 2004 Accepted 31 January 2005

PDB Reference: 15A9PPL-L-Ala complex, 1wc7. 


\section{research papers}

Table 1

Data-collection statistics for the Fab 15A9-PPL-L-Ala complex in three different space groups.

Values in parentheses are for the outer resolution shell.

\begin{tabular}{llll}
\hline Space group & $P 1$ & $\begin{array}{l}P 2_{1} \text { (correct } \\
\text { space group) }\end{array}$ & $\begin{array}{l}P 222_{1} \text { (incorrect } \\
\text { space group) }\end{array}$ \\
\hline $\begin{array}{l}\text { Unit-cell parameters } \\
\quad(\AA)\end{array}$ & & \\
$\quad b(\AA)$ & 63.5 & 63.5 & 63.6 \\
$\quad c(\AA)$ & 79.4 & 81.7 & 79.4 \\
$\alpha\left(^{\circ}\right)$ & 81.5 & 79.3 & 81.7 \\
$\beta\left({ }^{\circ}\right)$ & 89.8 & 90 & 90 \\
$\gamma\left({ }^{\circ}\right)$ & 89.8 & 89.98 & 90 \\
Resolution & 89.9 & 90 & 90 \\
No. of observations & $40-2.4(2.44-2.4)$ & $40-2.3(2.34-2.3)$ & $40-2.2(2.24-2.2)$ \\
No. of unique reflections & 5274068 & 382096 & 391534 \\
Completeness & $97.7(96.5)$ & 34338 & 20015 \\
Mean $I / \sigma(I)$ & $7.3(1.9)$ & $99.8(99.9)$ & $100(100)$ \\
$R_{\text {sym }}$ & $0.095(0.445)$ & $0.125(0.696)$ & $10.1(3.34)$ \\
$I>2 \sigma(I)(\%)$ & $81.8(44.2)$ & $81.6(42.1)$ & $86.159(0.64)$ \\
Redundancy & $1.86(1.82)$ & $3.31(3.09)$ & $6.34(6.0)$ \\
\hline
\end{tabular}

(Larsen et al., 2002; Barends \& Dijkstra, 2003). We report here the structure determination and successful refinement of another case of a pseudomerohedrally twinned monoclinic crystal form.

\section{Materials and methods}

\subsection{Protein purification}

The catalytic antibody 15A9 (Gramatikova \& Christen, 1996, 1997; Mouratou et al., 2000) was produced in ascitic fluid from hybridoma cells by the Bioatlantic company (taken over by the Laboratoire de Biotechnologies de l'Etablissement Français du Sang de Nantes). After ammonium sulfate precipitation, the $\operatorname{IgG}(\operatorname{IgG} 1, \kappa)$ was loaded onto a protein A column in $0.1 \mathrm{M}$ Tris- $\mathrm{HCl} \mathrm{pH} 8$ and eluted with $0.1 \mathrm{M}$ sodium citrate $\mathrm{pH}$ 3.8. The Fab was generated by overnight papain digestion of the antibody at $310 \mathrm{~K}$ under standard conditions (30 $\mathrm{m} M$ Tris- $\mathrm{HCl} \mathrm{pH} 7.4,138 \mathrm{~m} M \mathrm{NaCl}, 1.25 \mathrm{~m} M$ EDTA, $1.5 \mathrm{~m} M$ 2-mercaptoethanol) using a $6 \%(w / w)$ papain-to-antibody ratio. Undigested $\mathrm{IgG}$ and $\mathrm{Fc}$ fragments were removed by DEAE anion-exchange chromatography followed by gel filtration on a Sephacryl S100 HR column. The Fab was further purified by two steps of FPLC ion-exchange chromatography (Mono-S at $1 \mathrm{ml} \mathrm{min}^{-1}$ with a $30 \mathrm{ml}$ linear gradient of $0-0.25 \mathrm{M} \mathrm{NaCl}$ in $50 \mathrm{~m} M$ HEPES buffer $\mathrm{pH} 7.8$ then Mono$\mathrm{Q}$ at $1 \mathrm{ml} \mathrm{min}^{-1}$ with a $30 \mathrm{ml}$ linear gradient of $0-0.25 \mathrm{M} \mathrm{NaCl}$ in $20 \mathrm{~m} M$ ethanolamine buffer $\mathrm{pH} 9.35$ ).

\subsection{Crystallization}

Fab $15 \mathrm{~A} 9$ at $10 \mathrm{mg} \mathrm{ml}^{-1}$ was crystallized by the hangingdrop procedure by mixing $2 \mu$ protein solution containing PPL-L-alanine at a 1:1 molar ratio with $2 \mu \mathrm{l}$ of a $1 \mathrm{ml}$ reservoir solution containing $0.2 \mathrm{M}$ sodium iodide, $50 \mathrm{mM}$ sodium acetate $\mathrm{pH} 6,30 \%$ PEG 3350 . The presence of iodide was essential for crystallization. Crystals were flash-frozen in a nitrogen stream at $100 \mathrm{~K}$ in the same solution containing $15 \%$ glycerol.

\subsection{Data collection and analysis}

A data set to $2.3 \AA$ resolution (Table 1 ) was collected on beamline ID14-3 at the European Synchrotron Radiation Facility in Grenoble and processed with DENZO and SCALEPACK (Otwinowski \& Minor, 1997). The Stanley factor $\left\langle I^{2}\right\rangle /\langle I \mid\rangle^{2}$ and the Wilson's diffraction distribution were obtained from TRUNCATE (Collaborative Computational Project, Number 4, 1994).

\subsection{Molecular replacement and refinement}

Crystal twinning was not suspected initially and molecular replacement was carried out on the twinned data with $A M o R e$ (Navaza, 1994) using the atomic coordinates of the 15A9 antibody in complex with the hapten (PDB code 1wcb). In principle, crystal twinning should not hinder structure determination by molecular replacement (Yeates, 1997; Breyer et al., 1999). Both $P 2_{1}$ and $P 222_{1}$ space groups were tested. Although a plausible solution for the molecular replacement was found in $P 222_{1}$, it was abandoned because of numerous bad contacts. Self-rotation function analysis of the data processed in $P 2_{1}$ using $A M o R e$ showed the presence of a twofold axis perpendicular to the crystallographic $b$ axis, indicating possible higher (orthorhombic) space-group symmetry or the presence of a non-crystallographic axis perpendicular to, but not necessarily intersecting, the unique $b$ axis. The correlation coefficient was $96 \%$ in the resolution range $15.0-3.5 \AA$ with a Patterson integration radius of $40 \AA$.

The structure was initially refined in $C N S$ with the twinned data using NCS restraints. However, the refinement converged at unacceptable $R$ values: $R_{\text {cryst }}=31.1 \%, R_{\text {free }}=36.9 \%$. Refinement against intensities was continued using SHELX 97 (Sheldrick \& Schneider, 1997) including the twinning operator $(h,-k,-l)(\mathrm{TWIN})$, the twin fraction (BASF, initially arbitrarily set to 0.35 ) and NCS restraints (NCSY). To calculate $R_{\text {free }}$, the test reflections were selected in thin resolution shells; to avoid possible correlations introduced by the twinning, all the twin-related pairs belonged to either the test set or the reference set. The final refined structure has twinned $R_{\text {cryst }}=22.3 \%$ and $R_{\text {free }}=30.9 \%$ and a final twin fraction of 0.46 .

\section{Results and discussion}

\subsection{Detection of twinning}

Indexing and integration were possible in both primitive monoclinic $(P 2)$ or orthorhombic groups $(P 222)$, leading to unit-cell parameters $a=63.5, b=81.7, c=79.3 \AA, \alpha=\gamma=90$, $\beta=89.98^{\circ}$ or $a=63.6, b=79.4, c=81.7 \AA, \alpha=\beta=\gamma=90^{\circ}$, respectively (Table 1 ). The mosaicity refined to $0.86^{\circ}$. The presence of a twofold screw axis was established by analysis of the systematically absent reflections from data processed in $P 2$, leaving $P 2_{1}$ and $P 222_{1}$ as possible space groups. During post-refinement of unit-cell parameters, $\beta$ refined to $89.98^{\circ}$ in a monoclinic lattice and to $89.92^{\circ}$ in a triclinic lattice where no restrictions were applied to any of the unit-cell parameters. The $90^{\circ}$ value of $\beta$, the apparent $P 222$ symmetry (as witnessed 
by the relatively low merging $R$ factor in $P 222$ and the selfrotation function results), the split reflections in the diffraction pattern and the fact that the structure could not be satisfactorily refined raised the possibility that the crystal was twinned by the twinning operator $(h,-k,-l)$.

Twinning is often detected by an analysis of the second moment of intensities of acentric data $\left\langle I^{2}\right\rangle /\langle|I|\rangle^{2}$ (Yeates, 1997; Stanley, 1972). A value of 2.0 is expected in the absence of twinning and of 1.5 in the case of a perfect twin. The results of the second moment of intensities of acentric data for the 15A9-PPL-L-Ala data were examined not only for the whole resolution range but also for a high-resolution range, as the effects of twinning may not be manifested at low resolution for a pseudo- $P 222_{1}$ crystal. However, the calculated value of 2.04 for data between 40 and $2.3 \AA$, and 2.06 for data between 3 and $2.5 \AA$ suggested no twinning at all. Moreover, the observed cumulative intensity distribution $N(z)=f(z)$ for acentric data was only slightly sigmoidal (Fig. $1 a$ ), indicating a low degree of twinning at most. Two other twinning tests were then performed. Firstly, $\langle|F|\rangle^{2} /\left\langle F^{2}\right\rangle$ for acentric data, which should be 0.785 for untwinned and 0.865 for perfectly twinned data, was calculated in CNS. For 15A9-PPL-L-Ala it is 0.819, pointing to the presence of partial twinning with a high twinning fraction. A discrepancy between the two twinning indicators $\left\langle I^{2}\right\rangle /\langle|I|\rangle^{2}$ and $\langle|F|\rangle^{2} /\left\langle F^{2}\right\rangle$ has also been reported in a similar case with pseudotranslation (Rudolph et al., 2004). Indeed, it is known that the Stanley factor $\left\langle I^{2}\right\rangle /\langle|I|\rangle^{2}$ can behave abnormally and a newer test based on local intensity differences that is insensitive to anisotropy, pseudo-centring or
Table 2

Refinement statistics for the Fab 15A9-PPL-L-Ala complex.

\begin{tabular}{ll}
\hline Twin fraction & 0.46 \\
Protein atoms & 6465 \\
Water molecules & 120 \\
Ligand molecules & 1 \\
Iodide ions & 5 \\
Resolution & $20-2.3$ \\
$R_{\text {free }}(5 \%)$, all data & 0.308 \\
$R$, all data & 0.223 \\
$R_{\text {free }}(5 \%), F>4 \sigma(F)$ data & 0.277 \\
$R, F>4 \sigma(F)$ data & 0.201 \\
Bond-length deviation $(\AA)$ & 0.004 \\
Bond-angle deviation $\left({ }^{\circ}\right)$ & 1.58 \\
Ramachandran plot, $\%$ residing in & \\
$\quad$ Core regions & 77.6 \\
$\quad$ Additional allowed regions & 21.8 \\
$\quad$ Disallowed regions & 0.5 \\
Average $B$ value of protein atoms $\left(\AA^{2}\right)$ & 48.1 \\
Average $B$ value of water molecules $\left(\AA^{2}\right)$ & 40.2 \\
Average $B$ value of iodide ions $\left(\AA^{2}\right)$ & 77.4 \\
Average $B$ value of ligand $\left(\AA^{2}\right)$ & 41.5 \\
\hline
\end{tabular}

pseudo-non-crystallographic symmetry has been implemented (Padilla \& Yeates, 2003). This test was performed for the 15A9-PPL-L-Ala data set using DATAMAN (v.6.3 or above, PY_stats command) from the $R A V E$ suite (Uppsala Software Factory). The cumulative probability distribution $N(|L|)$ versus $|L|$, where $L$ is defined as $\left[I\left(h_{1}\right)-I\left(h_{2}\right)\right] /\left[I\left(h_{1}\right)+I\left(h_{2}\right)\right]$ and $I\left(h_{1}\right)$ and $I\left(h_{2}\right)$ are the intensities of the unrelated reflections $h_{1}$ and $h_{2}$, indeed gave a clear indication of twinning (Fig. 1b). The observed values of $\langle|L|\rangle$ and $\left\langle L^{2}\right\rangle$ were 0.44 and 0.268 , respectively, which are between the theoretical

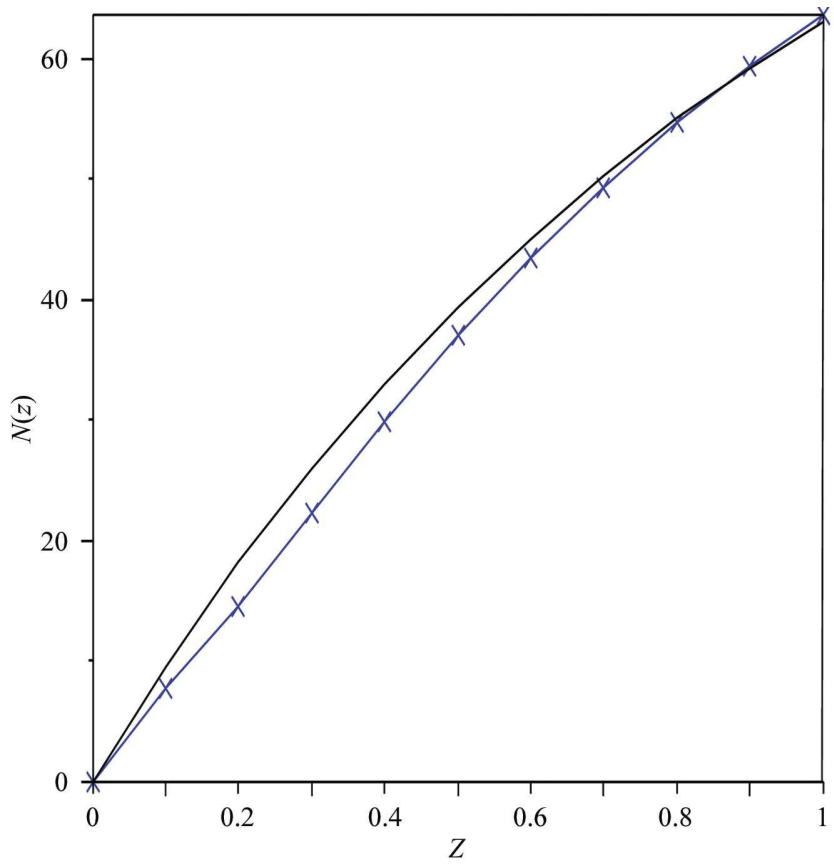

(a)

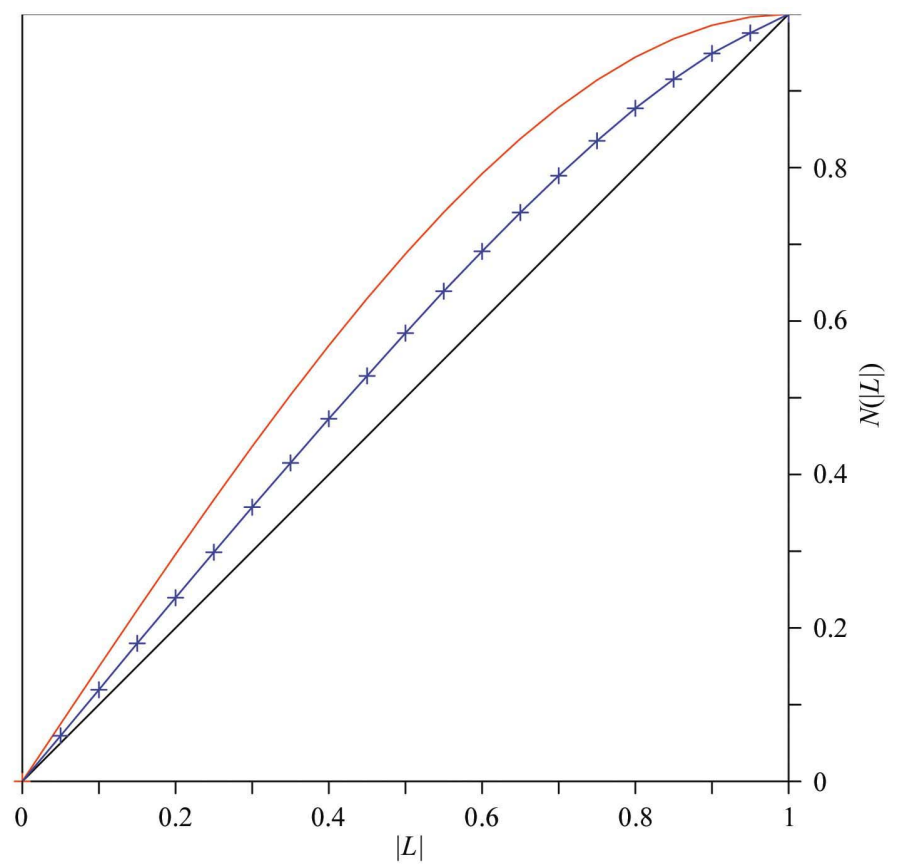

(b)

Figure 1

Cumulative intensity distribution for the Fab15A9-PPL-L-Ala data set. Theoretical distributions for untwinned and perfectly twinned acentric data are shown by black or red curves, respectively. Distributions for observed acentric data are shown by blue line and crosses. ( $a$ ) The traditional intensity statistics curve, $N(z)$ versus $z$, where $z=I /\langle I\rangle$, gives no clear indication of twinning. $(b)$ The local intensity statistics curve, $N(|L|)$ versus $|L|$, where $L=\left[I\left(h_{1}\right)-I\left(h_{2}\right)\right] /\left[I\left(h_{1}\right)+I\left(h_{2}\right)\right]$ and $I\left(h_{1}\right)$ and $I\left(h_{2}\right)$ are the intensities of the unrelated reflections $h_{1}$ and $h_{2}$, falls between the curves expected for untwinned and perfectly twinned data. 
values for acentric perfectly twinned data ( 0.375 and 0.2 , respectively) and those for acentric twinned data $(0.5$ and 0.333 , respectively; Padilla \& Yeates, 2003).

\subsection{Determination of the twin fraction}

The twin-fraction determination tests performed by XPREP, CNS or the twinning server (http://www.doe-mbi.ucla.edu/ Services/Twinning/; Yeates, 1997) are not allowed for a monoclinic space group with pseudomerohedral twinning. Therefore, the twin fraction was estimated with SHELX. The refined high twinning fraction of 0.46 is consistent with the low $R_{\text {merge }}$ value obtained in space group $P 222_{1}$. Detwinning of intensities is not recommended with such a high twinning fraction.

\subsection{Refinement against twinned data}

The structure was therefore successfully refined against the originally measured intensities in SHELX, a program that can directly refine against twinned data (Table 2). This definitely established that the true space group is $P 2_{1}$ and that the data are pseudomerohedrally twinned. In the two other examples of twinning in a monoclinic space group with $\beta=90^{\circ}$, the NCS rotational operator is parallel to the twinning operator (Larsen et al., 2002; Barends \& Dijkstra, 2003). For 15A9, this is also the case; the twinning operator is a twofold axis along the $a$ axis, while the NCS operator is a 2 screw axis nearly parallel to the $a$ axis coupled to a translation of approximately $(-0.25,0.47,0.56)$.

\subsection{Model analysis}

The structure of the PLP-dependent catalytic antibody 15A9 complexed with phosphopyridoxyl-L-alanine (PPL-L-Ala), shows that the substrate analogue is buried in a shallow cleft (Figs. $2 a$ and $2 b$ ). This was expected as the position preceding H101 is occupied by a bulky residue (Phe H100f in 15A9; Mouratou et al., 2000; GolinelliPimpaneau, 2000). Antibody 15A9 has a phosphate-binding pocket similar to that of 28B4, another catalytic antibody possessing a bulky residue at position H100c (preceding H101) that has been raised against an aminophosphonic hapten (Hsieh-Wilson et al., 1996) (Fig. 2c).

Five iodide ions were found in the 15A9-PPL-L-Ala structure. While one of the two combining sites is occupied by one PPL-L-alanine molecule (Figs. $2 a$ and $2 b$ ), one iodide ion

Figure 2
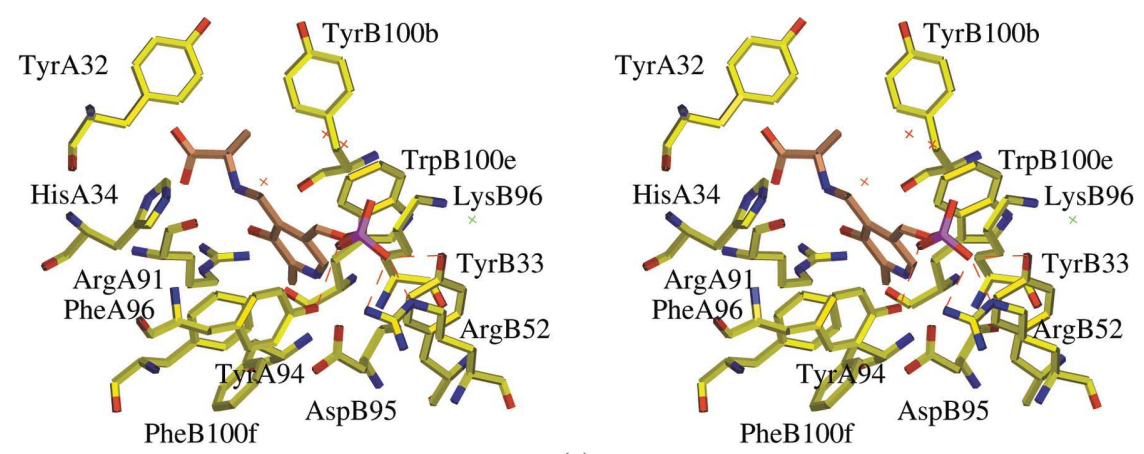

(a)
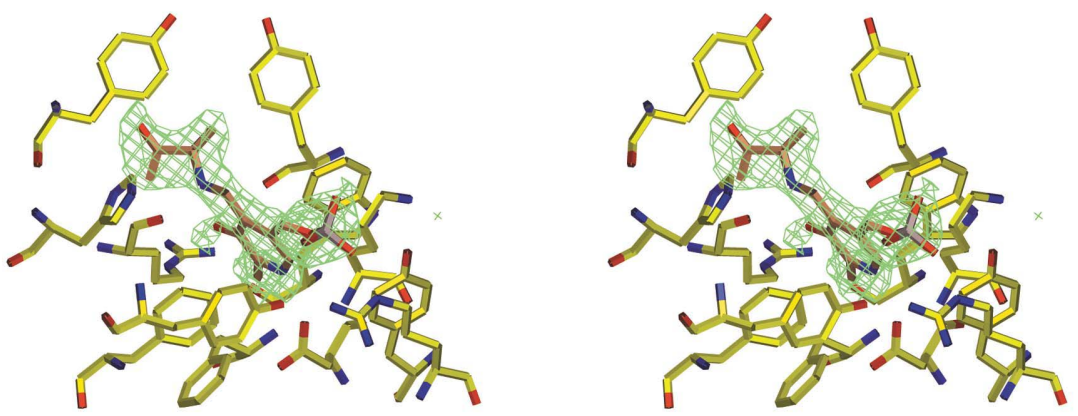

(b)
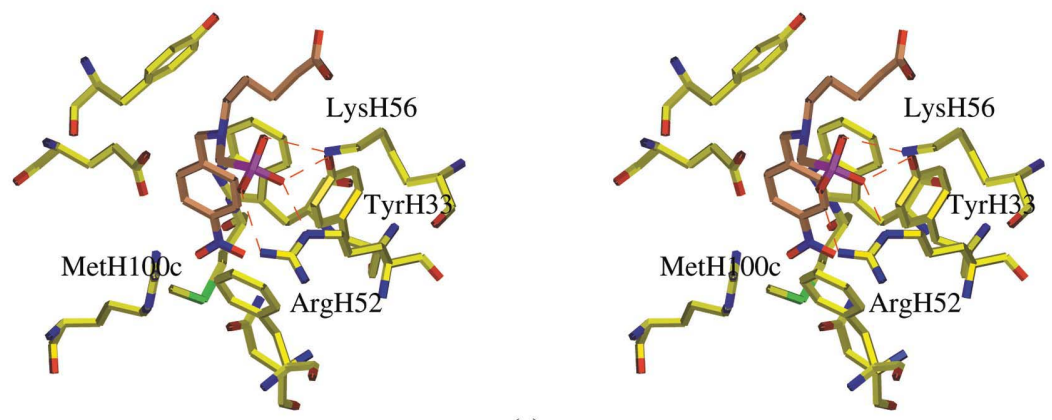

(c)

Structure of the combining site of antibody 15A9 in complex with PPL-L-Ala. The atom colouring is as follows: $\mathrm{C}$ atoms of the proteins are in yellow, $\mathrm{C}$ atoms of the ligand in $\tan , \mathrm{O}$ atoms in red, $\mathrm{N}$ atoms in blue and $\mathrm{P}$ atoms in magenta. Water and iodide ions are indicated as red or green crosses, respectively, and hydrogen bonds as red dashed lines. (a) Stereoview of the PPL-L-Ala ligand in the active site of Fab 15A9. The residues (TyrL32, HisL34, ArgL91, TyrL94, PheL96, TyrH33, ArgH52, AspH95, LysH96, TyrH100b, TrpH100e) that interact with the substrate analogue have been represented. The $\mathrm{C} 5-\mathrm{C} 4-\mathrm{C} 4 \mathrm{~A}-\mathrm{N}$ dihedral angle deviates from planarity by $4^{\circ}$ and the $\mathrm{C} 4-\mathrm{C} 4 \mathrm{~A}-\mathrm{N}-\mathrm{C}^{\alpha}$ angle by $29^{\circ}$. The phosphate group of the cofactor hydrogen bonds to TyrH33 and TyrL94 and is involved in an ionic interaction with ArgH52. (b) A $\sigma_{A}$-weighted $F_{\text {obs }}-F_{\text {calc }}$ electron-density map omitting the ligand is contoured at the level of $1 \sigma$ and is superimposed on the active-site structure. For the calculation of electron-density maps, SHELXL partitions the observed intensity in the ratio of the calculated contributions, i.e. $F_{\text {obs }}^{2}($ total $)=F_{\text {calc }}^{2}($ total $) \times F_{\text {obs }}^{2}($ twin 1$) / F_{\text {calc }}^{2}($ twin 1$)$, where the $F_{\text {calc }}$ values include the twin fraction. (c) Stereoview of the active site of Fab 28B4. Antibody 28B4 catalyzes the periodatedependent oxidation of sulfite (Hsieh-Wilson et al., 1996). The bottom of the cavity is occupied by the bulky residue MetH100c and the hapten binds in a shallow pocket at the upper part of the VL-VH interface. The phosphate group of the aminophosphonic hapten hydrogen binds to TyrH33 and is involved in an ionic interaction with ArgH52 and LysH56.

mimics the PLP phosphate in the other (Fig. 3a). Moreover, one iodide ion fixes the conformation of CDRH3 in each molecule of the asymmetric unit (Fig. $3 b$ ). The last two iodide molecules are found at the interface between two symmetryrelated molecules or between the two molecules of the asymmetric unit (Fig. 3c). While the iodide ion that takes the place of the phosphate group is involved in an electrostatic 


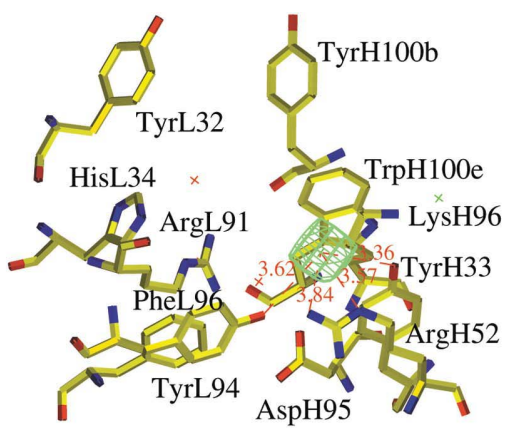

(a)
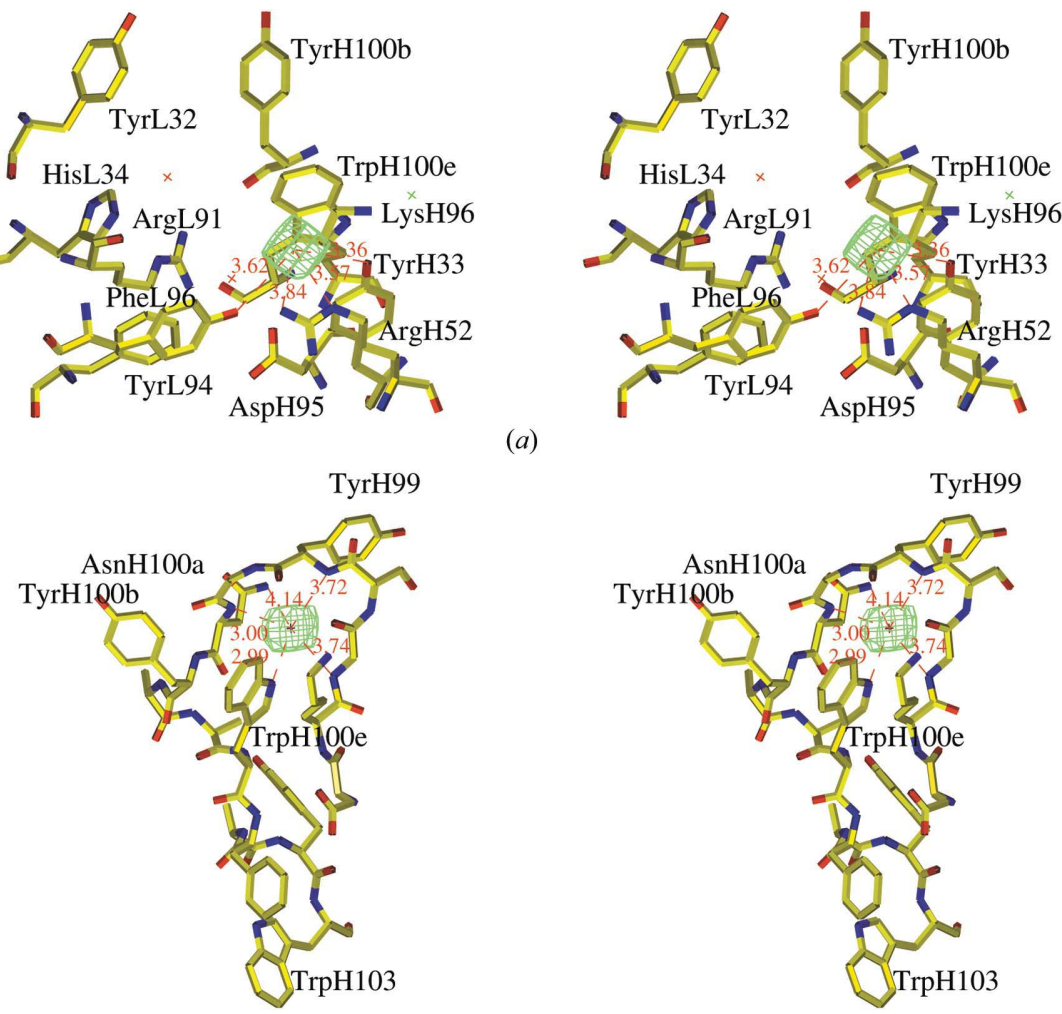

(b)
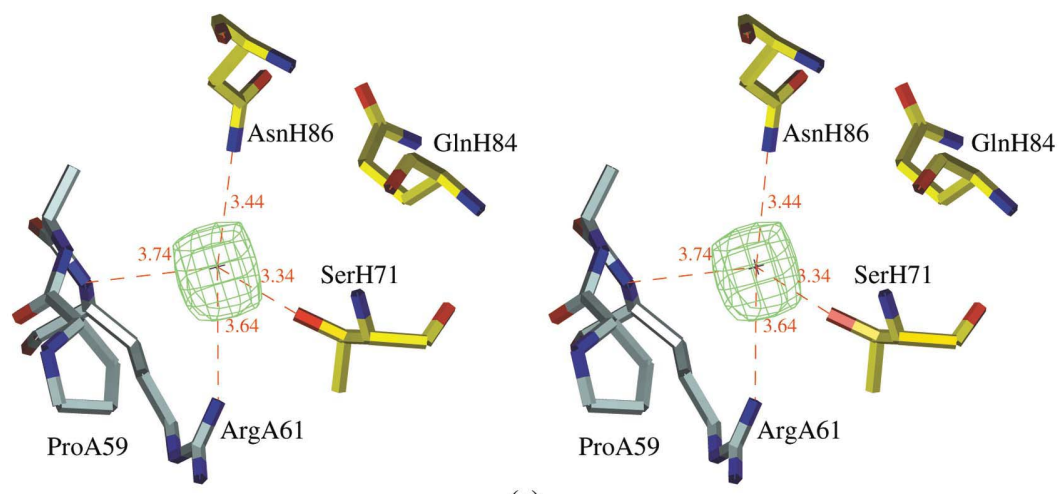

(c)

Figure 3

The iodide ions in the Fab15A9-PPL-L-Ala structure. A $\sigma_{A}$-weighted $F_{\text {obs }}-F_{\text {calc }}$ electrondensity map omitting the iodide ions is contoured at the level of $2 \sigma$ and superimposed on the active-site structures. (a) Stereoview of the non-occupied active site of the Fab15A9-PPL-LAla complex. One iodide ion takes the place of the phosphate group in one molecule of the asymmetric unit. (b) The conformation of CDRH3 is stabilized by an iodide ion. In each molecule of the asymmetric unit, one iodide ion makes four 3.0-3.8 $\AA$ interactions with the $\mathrm{NH}$ groups of His97 and His99, TrpH100e $\mathrm{N}^{\varepsilon 1}$ and AsnH100a $\mathrm{N}^{\delta 2}$. (c) One iodide ion interacts with the two molecules of the asymmetric unit. At the corresponding position in the other molecule, one iodide ion is involved in a crystallographic contact.

interaction with $\mathrm{ArgH} 52$ and contacts tyrosine residues (Fig. $3 a$ ), the environment of the other iodide ions more classically (Vaney et al., 2001) involves mostly $\mathrm{N}$ atoms: amide $\mathrm{N}$ atoms from the main chain and/or the $\mathrm{N}$ atom from an asparagine, arginine or tryptophan side chain (Figs. $3 b$ and $3 c$ ).
The comparison of the structure of the 15A9-PPL-L-Ala complex described here with that of the 15A9-PPL-D-Ala and 15A9PPL-L-Lys complexes together with a plausible mechanism of the reaction will be published elsewhere.

I thank Philipp Christen for the gift of phosphopyridoxyl-L-alanine and of hybridoma cells of 15A9 and for very useful discussions and Thierry Bizebard, Marcel Knossow, Roger Fourme and Thierry Prangé for reading the manuscript. The cost of the ascites production was covered by the Centre National de la Recherche Scientifique, Groupement de Recherche 897.

\section{References}

Barends, T. R. M. \& Dijkstra, B. W. (2003). Acta Cryst. D59, 2237-2241.

Breyer, W. A., Kingston, R. L., Anderson, B. F. \& Baker, E. N. (1999). Acta Cryst. D55, 129-138.

Collaborative Computational Project, Number 4 (1994). Acta Cryst. D50, 760-763.

Declercq, J.-P. \& Evrard, C. (2001). Acta Cryst. D57, 1829-1835.

Golinelli-Pimpaneau, B. (2000). Curr. Opin. Struct. Biol. 10, 697-708.

Gramatikova, S. I. \& Christen, P. (1996). J. Biol. Chem. 271, 30583-30586.

Gramatikova, S. I. \& Christen, P. (1997). J. Biol. Chem. 272, 9779-9784.

Hsieh-Wilson, L. C., Schultz, P. G. \& Stevens, R. C. (1996). Proc. Natl Acad. Sci. USA, 93, 53635367.

Larsen, N. A., Heine, A., de Prada, P., Redwan, E.-R., Yeates, T. O., Landry, D. W. \& Wilson, I. A. (2002). Acta Cryst. D58, 2055-2059.

Mouratou, B., Gramatikova, S. I., Kunz, B. \& Christen, P. (2000). Mol. Immunol. 37, 633-640.

Navaza, J. (1994). Acta Cryst. A50, 157-163.

Otwinowski, Z. \& Minor, W. (1997). Methods Enzymol. 276, 307-325.

Padilla, J. E. \& Yeates, T. O. (2003). Acta Cryst. D59, 1124-1130.

Rudolph, M. G., Wingren, C., Crowley, M. P., Chien, Y.-H. \& Wilson, I. A. (2004). Acta Cryst. D60, 656-664.

Sheldrick, G. M. \& Schneider, T. R. (1997). Methods Enzymol. 277, 319-343.

Stanley, E. (1972). J. Appl. Cryst. 5, 191-194.

Vaney, M. C., Broutin, I., Retailleau, P., Douangamath, A., Lafont, S., Hamiaux, C., Prangé, T., Ducruix, A. \& Riès-Kautt, M. (2001). Acta Cryst. D57, 929-940.

Yang, F., Dauter, Z. \& Wlodawer, A. (2000). Acta Cryst. D56, 959964.

Yeates, T. O. (1997). Methods Enzymol. 276, 344-358. 